\title{
Sur la traduction du théâtre francophone africain : l'exemple de Werewere Liking
}

\author{
Nataša Raschi \\ Università degli Studi di Perugia
}

Toute pièce, conçue pour un public donné, évoque des contextes - littéraires, sociaux, moraux, historiques et géographiques - ainsi que des situations d'une grande précision (Mounin 90). JeanPierre Vernant et Pierre Vidal-Naquet affirment que «le contexte (...) ne se situe pas au marge des œuvres, (...) il constitue un sous-texte qu'une lecture savante doit déchiffrer dans l'épaisseur même de l'œuvre » (Vernant, Vidal-Naquet 23). La traduction d'une œuvre théâtrale peut donc exalter la valeur d'éléments extrêmement polyvalents et présents dans chaque passage du texte. Du point de vue linguistique, les écrivains francophones glissent souvent des variantes ou des tournures liées à leur univers d'appartenance. Il s'agit d'idiomes parfois très proches du français, parfois complètement différents, qui ont évolué pendant un certain temps et qui ont parfois atteint leur autonomie selon le milieu, les vicissitudes, les contacts et les interférences subies.

La plupart des productions littéraires de l'Afrique francophone fourmille de mots et d'expressions empruntés aux langues ethniques. Dans bien des cas, nous avons la possibilité de nous adresser directement à l'auteur pour dissiper tout doute, mais dans le cas de ceux qui ont disparu, il faut toujours chercher des textes parallèles, contemporains ou écrits par lui-même ou encore issus de la même région, d'une même philosophie ou d'un parcours de recherche identique. Étant donné qu'une pièce se caractérise par l'alternance de moments statiques et dynamiques, et que pour cette raison sa particularité est liée au rythme, nous avons essayé de traduire de tels ouvrages sans en appauvrir la richesse variée et multiforme, et cela dans le but de garder le rythme original. Le rythme est considéré en fait comme une recherche de précision et d'harmonie, ou, comme le souligne Henri Meschonnic, «si le rythme est dans le langage, dans un discours, il est une organisation (disposition, figuration) du discours, (...) [une] organisation du sens dans le discours » (Meschonnic Critique 70).

A ce propos, nous aimerions concentrer notre attention sur la dernière production de Werewere Liking - Le Parler-Chanter et Médée - que nous avons traduit en italien. ${ }^{1}$ Auteur de pièces de théâtre, d'essais et de romans, mais aussi metteur en scène, peintre et écrivain, elle est originaire du Cameroun et, en 1985, fonde à Abidjan (Côte d'Ivoire) un groupe de création artistique. Ce dernier réunit écrivains, acteurs, musiciens, plasticiens et autres venus de tous les coins d'Afrique : ensemble, ils animent la communauté du Village Ki-Yi. S'agissant d'œuvres créées en vue de la représentation scénique, plus aptes à être vues que lues, nous essayerons également d'en découvrir les traits essentiels du texte-source (Ladmiral 33) ainsi que des résultats donnés par la traduction.

Ainsi, nous allons nous concentrer sur une pratique de mise en commun, d'échange et de discussion qui conduit à la réinterrogation des traditions, à la réinterprétation des cultures anciennes et à de nouvelles formes d'expérimentation. Dans de telles situations, le traducteur doit envisager plusieurs procédures, en plus de suivre naturellement des règles de bon sens. Si, par exemple, nous choisissons de ne pas traduire les expressions en langue ethnique pour notre

\footnotetext{
1 En particulier, il s'agit de Le Parler-Chanter, Parlare cantando. Torino: L'Harmattan Italia, 2003 (Introduction, Traduction et Note sur la traduction de Nataša Raschi) et de Médée les risques d'une réputation / Medea i rischi d'una certa reputazione. Ed. Anna Paola Mossetto, Torino: Libreria Stampatori, 2006 (Traduction, Note sur la traduction et interview à l'auteur de Nataša Raschi).
} 
public-cible, nous pourrions opter pour des notes en bas de page ou pour un glossaire explicatif. Ces deux solutions sont valables pour faciliter la lecture et pour arriver à saisir véritablement les caractéristiques profondes du texte original.

\section{Le Parler-Chanter ${ }^{2}$}

Habile jongleur de sons et de sens, Werewere-Liking harmonise ici avec une rare vigueur les éléments culturels les plus éloignés : la valeur spirituelle des masques et des rituels traditionnels, unie à l'attrait pour quelques aspects de la modernité, produit un admirable mélange. L'on y remarque des images qui remontent aux racines les plus profondes du patrimoine culturel africain et de son imaginaire merveilleux, notamment aux contes de l'oralité dans lesquels tout élément animaux, chiffres, instruments - s'enrichit d'une valeur connotative.

Dans La Veuve Diyilèm se juxtaposent la voix d'une jeune veuve et la voix off de son mari récemment décédé. Après avoir accusé sa femme d'avoir de nombreux amants, en réalité - ainsi qu'il l'a écrit dans une lettre - la faute lui appartient complètement ; c'est lui qui l'a trompée et qui a contracté le virus du SIDA. Les insertions de fragments de la lettre, qui seule demeure pour combler leurs incompréhensions, permettent à la femme de saisir ce que son mari n'arrivait vraiment pas à tolérer chez elle : son indépendance. Et c'est justement à cause de ce manque de dialogue que la confrontation a lieu trop tard: le mari ne s'est pas libéré de ses doutes et la femme ne s'est jamais entièrement dévoilée.

L'enfant Mbénè est un conte initiatique construit dans le respect de la tradition orale. L'on y reconnaît aisément les rituels dont parle Julienne Froelich à propos des sociétés d'initiation et dont le but est de « (...) transformer les adolescents en citoyens, membres efficaces de la société, et [de] intégrer les individus dans la communauté des vivants et des morts ; les rites confirment leur accession à la vie sociale du groupe. » (129). La pièce s'ouvre sur le désespoir d'un homme veuf qui se retrouve seul avec son fils unique Mbénè, dont le nom en bassa, idiome du Cameroun méridional d'où l'auteur est originaire, signifie «bizarre ». Tout le village protège exagérément le jeune homme, jusqu'à ce que son père ne décide de lui donner une leçon de courage. Les entreprises auxquelles il est obligé de se soumettre sont dangereuses, mais grâce à son humilité, à son sens du devoir et de la justice, Mbénè arrive à franchir toutes les étapes de son parcours initiatique.

Un véritable foisonnement de symboles (couleurs, chiffres, animaux) prend place devant nos yeux. Les marionnettes surtout sont toujours présentes dans les spectacles de Werewere Liking qui a consacré une étude approfondie à celles du Mali ${ }^{3}$. Leur intérêt réside véritablement dans ce jeu de miroirs entre la créature et son double incessamment adressé au spectateur, comme s'il s'agissait d'objets de bois qui s'animent à une existence éphémère avant de revenir à leur immobilisme originaire. Ces ouvrages prévoient aussi l'insertion de chants, de musiques (jouées par des instruments traditionnels et modernes à la fois), de danses et de chœurs qui ponctuent l'action. Dans le respect de la tradition africaine, la musique est ici tellement liée au texte qu'elle «n'est pas mélodie mais message » (Pacéré 87). Chez l'artiste camerounaise, musique signifie alors rencontre et mouvement, épopée et initiation. Tout a lieu comme si le seul fait de pouvoir

\footnotetext{
2 Les deux pièces traduites en ce volume s'insèrent dans l'avant-dernière production dramaturgique de Werewere Liking, des inédits dont l'auteur nous avait fait cadeau. Cette publication ne concerne pourtant que deux pièces parmi d'autres qui nous avaient été données. Au total, il s'agissait de La Queue du Diable, deuxième version, 1996,25 p. dactylographiées ; La Veuve Diyilèm (Dilemme), sans date, 15 p. dact. ; Héros d'eau, s.d., 23 p. dact. ; Quelque chose Afrique, s.d., 22 p. dact. ; L'Enfant Mbénè, 1997, 17 p. dact. Ces cinq pièces composent Le Parler-Chanter.

${ }^{3}$ Lire à ce propos Liking, Werewere. Marionnettes du Mali. Paris : NEA-Arhis, 1987.
} 
disposer d'un auditoire permettait à de telles pièces d'exister et de transmettre aux artistes l'énergie nécessaire à poursuivre dans le spectacle.

Cet effet de participation se retrouve surtout dans L'enfant Mbénè : les conteurs toujours présents sur scène rappellent constamment l'attention du public et sont à leur tour interrogés par le jeune initié à signifier la perpétuité du message et de l'enseignement donné, ce qui constitue aussi le but dernier de la narration des contes. L'auteur attribue aussi une grande importance à un personnage nouveau : M.S., le Metteur en Scène, dont les interventions non seulement scandent et rythment les moments cruciaux de l'action, mais lui donnent en même temps une forte impulsion. C'est ainsi que l'effet de théâtre dans le théâtre est parfaitement atteint.

Ces deux pièces de Werewere Liking nous présentent un monde riche en personnages et en valeurs très différentes de celles de l'Occident. Le style kaléidoscopique de l'ouvrage ainsi que de sa mise en scène embrassent le multiculturalisme et les thèmes ethno-anthropologiques. Nous nous sommes interrogée sur la démarche la plus adéquate vis-à-vis des tournures typiquement africaines et nous avons choisi de transcrire les notes rédigées par l'auteur, source - il faut le souligner - de nombreux conseils précieux. Il convient de remarquer que ces formes, particulièrement aliénantes quant à leur effet, n'avaient pas un caractère typographique spécial dans le texte source et que nous avons maintenu le choix de l'original. Même si on ne peut parler de pluralisme linguistique, nous pouvons expliquer ces expressions en langues ethniques que par la multitude des personnages présents, venus de divers horizons géographiques. Selon la philosophie sous-jacente à la création du Village $K y-Y i$, les mots qui appartiennent au bagage culturel des différents peuples représentés doivent se fondre et se confondre pour créer des pièces-mosaïques.

En lisant ces œuvres, nous avons rencontré «Gbèlèya » qui en dioula - langue des commerçants et des marchands du Nord de la Côte d'Ivoire - signifie « pauvreté, problèmes »; «Aboloo » qui en noutchi - «le produit de toute cette masse de désœuvrés, de déscolarisés, jetés à la rue, qui fabriquent un code langagier, relevant d'un certain savoir linguistique » (Sidibe 98) - indique la grandeur et la solidité ; "Guzông » qui pour les Bétés - peuple vivant dans le Centre-Ouest de la Côte d'Ivoire - évoque un tamis filtrant à gros trous pour séparer le composé liquide des résidus de noix de palme pour la préparation de la sauce graine, célèbre plat national. Du Cameroun, viennent «Ekkié», exclamation de surprise ou d'exaspération en béti, et l'expression « $A$ mbong mè magnii magnii », qui veut dire «ça me dégoûte », propre au registre populaire.

Il serait, en revanche, très compliqué de remonter aux sources du nom donné au Clavier narrateur, car son origine est multiple: Dinoo (doigts), Makôô (pieds), Ngognoo (mille-pattes), Nkôt (musicien), Nganda (guitare bassa à cinq cordes), Ndangui (balafon bassa). Tout cela pour mettre en relief la maitrise aussi bien que la rapidité des doigts jouant du clavier, de la guitare, du balafon, par rapport justement aux pattes agiles des mille-pattes. Puisque les personnages expliquent immédiatement les termes inconnus de leurs racines culturelles, nous nous sommes résolue à garder les expressions africaines même dans le texte en regard et donc à ne pas introduire de commentaire explicatif.

Dans le respect de l'original, nous ne sommes pas intervenue sur les tournures où le signifiant l'emporte, c'est-à-dire sur celles qui sont prononcées pour éveiller l'attention du public sur l'action en cours. Suivant une technique ancienne, Anguinguilayé est une invocation que le narrateur adresse à l'auditoire pour lui demander s'il doit continuer ou non son récit. Le public 
répond Yessé en l'encourageant ainsi à poursuivre. ${ }^{4}$ Tout conte oral envisage des exclamations enthousiastes - «Didiga Zara!» s'écrient les Bétés -, plus importantes pour leur effet que pour leur contenu. Pour toutes ces raisons, et tout en sachant que Youyou indique les cris de joie poussés par les femmes et les enfants pour annoncer l'entrée sur scène de quelque personnage absent depuis longtemps, qu'Humébummm n'est qu'un son onomatopéique pour évoquer le passage de la sauce graine (célèbre assaisonnement ivoirien) à travers les trous du tamis et encore Tché tché tché est une exclamation de surprise, nous avons opté pour leur retranscription dans le texte cible. C'est ainsi que les assonances, les allitérations, les exhortations et les cris, concourent à la construction du sens.

Médée

Composée de huit scènes, cette Médée ${ }^{5}$ - portant le sous-titre Les risques d'une réputation -s'ouvre et se ferme sur un colloque de conteuses qui analysent à fond ce mythe. Werewere Liking - qui appuie son étude sur les versions d'Euripide, Sénèque et Christa Wolf - refuse la tradition qui voudrait qu'une mère soit meurtrière de ses propres enfants soit en vertu du respect fondamental envers la vie donnée, soit à cause des traditions polygamiques (Mossetto 387). Sept femmes venues des quatre coins du monde ont animé la scène du Teatro Baretti à Turin ${ }^{6}$ et leur rencontre s'est située surtout au niveau linguistique - français, guéré, napolitain, serbe - lorsque Médée décide de lancer une malédiction contre l'injustice qui voudrait l'écraser. Cela se manifeste sur scène parce que la parole, qui intervient au moment du danger, ne peut être que celle des origines, tandis qu'au niveau du texte écrit on trouve un chant en bassa suivi par son correspondant en langue française (Liking Médée 30-34). Multiforme et bigarrée, cette langue est parcourue à la fois par une pulsation de vitalité et d'éloignement.

Voilà pourquoi l'eau est l'élément fondamental que l'on associe immédiatement à cette version de Médée. Une eau inquiétante, par moments libératoire, mais jamais égale. D’après un proverbe africain, l'eau qui coule n'est jamais la même et c'est justement cette impression que suscite le texte : à chaque écoute, il transmet des émotions différentes et très fortes. Une eau qui est voyage et purification, comme cela se produit dans toutes les versions de Médée, mais qui est aussi renouvellement et richesse, car cette Médée se multiplie par trois pour rendre la complexité, la pluralité du monde des «barbares» (Tagliacozzo 133) qu'elle représente. Ainsi l'œuvre connaîtelle une "Médée Source», une "Médée Rivière» et une "Médée Fleuve», qui sont respectivement devenues «Medea Fonte», "Medea Affluente» et «Medea Fiume». Cette interprétation nous a volontairement permis de créer une sonorité commune, précieuse et fluide, dont le texte de départ est dépourvu. Medea Fonte transmettrait l'importance des origines et des racines, Medea Affluente renverrait à l'idée de croissance et de descendance, alors que Medea Fiume correspondrait au comble de la maturité, à la force et à la majesté. De telles présences se reflètent dans l'amplification de voix inquiétantes qui se succèdent avec un rythme pressant. La traduction prenait donc des airs de défi que nous avons décidé de relever.

Les voix coulent sans cesse comme de l'eau, mais l'omniprésence de cet élément n'empêche pas aux autres de se manifester. Une Médée de feu - enflammée par ces calomnies cruelles et «cette réputation » néfaste - qui, digne petite-fille du Soleil, est aussi capable d'écorcher ses ennemis. Une Médée de terre, fermement attachée à ceux qui ont tout laissé pour la suivre, à ceux pour lesquels elle a déployé ses efforts, à ces enfants qui ne se manifestent jamais sur scène, mais qui

\footnotetext{
${ }^{4}$ Ces deux expressions se répètent souvent dans le théâtre rituel, car le «meneur de rite » doit stimuler la participation du public.

5 Pour une lecture de la pièce : Cf. Mossetto, Anna Paola. "La sfida africana di Medea." Magia, gelosia, vendetta. Il mito di Medea nelle lettere francesi. Ed. Liana Nissim et Alessandra Preda. Milano : Cisalpino, 2006. 379-391.

${ }^{6}$ Werewere Liking, Médée, les risques d'une réputation, du 7 au 14 janvier 2005.
} 
sont pourtant tangibles dans les battements de son cœur de mère. Une Médée à la fois de terre et d'air, si l'on considère son attachement viscéral à sa nature rhizomatique, ce qui la pousse à invoquer le ciel aussi bien pour son passé mythique que pour les malédictions à lancer.

Cette Médée ressemble au jeu des poupées gigognes, mais son emboîtement présente des frontières flottantes qui s'amplifient et qui débordent en toute occasion. Tout comme la transposition continuelle du plan mythique à celui du séminaire sur le mythe : il s'agit encore une fois de femmes qui se mesurent avec Médée pour fouiller tous ses aspects, ou pour en laisser entrevoir d'autres, peut-être terribles, peut-être indicibles. Le jeu des renvois entre ce qui est dit et ce qui ne l'est pas doit pourtant être traduit. De surcroît, il faut accorder une attention constante aux sous-entendus qui parsèment la pièce et font toujours allusion aux questions cruciales de l'actualité, d'une humanité ni blanche ni noire, mais pleinement évoquée par la nuance sonore à mi-chemin entre les mots français «étranger» et «étrange», et les traductions italiennes «straniero » et « estraneo ».

Notre traduction s'est inspirée de la vision du spectacle de Turin, mais elle a tout de même exigé, d'un point de vue purement textuel, une analyse fouillée, pour résoudre certains points critiques et éclairer les passages obscurs. En outre, la traduction doit prendre en compte l'énorme production littéraire sur Médée et tous les parcours de lecture possibles pour maîtriser le contenu total à la disposition de l'auteur (Eco 90, 191). Dans un essai important, où il essaie de réfuter le lieu commun d'après lequel le critique littéraire serait le meilleur traducteur des textes de l'auteur dont il est spécialiste, Graziano Benelli en détruit un autre, peut-être plus enraciné, selon lequel traduire vers sa propre langue serait facile (Benelli 72, 74). C'est forte de ces affirmations que nous nous sommes mesurée à ce texte : une "pièce-poème » qui se rapproche des rimes, des assonances et des scansions musicales propres aux vers classiques. Puisque la prose alterne avec les octosyllabes et les dodécasyllabes, c'est la véhémence de la langue qui a le dessus et c'est justement cette ardeur que nous avons cherché à transposer dans la version italienne, tentant de respecter autant que possible l'original dans sa richesse alchimique, et totalement fidèle au concept cher à Buffoni (111) de la loyauté envers le texte source.

Etant donné la complexité de l'œuvre, les critères à suivre pour la traduction ne pouvaient qu'être multiples: en premier lieu, respecter scrupuleusement les tonalités du texte de départ, tout comme les pics d'intensité émotive liés à une syntaxe qui s'écarte souvent de la norme de l'ordre direct sujet-verbe-objet. Il importe de remarquer l'élégance de Werewere Liking, artiste multiforme et douée qui se distingue par ses tableaux magnifiques et son talent de musicienne raffinée. C'est justement de musique qu'il s'agit, si l'on considère même seulement les jeux de rimes et de sifflantes de la Scène 5: «Oui sans pouvoirs l'amour n'était que du vent / Et sans amour les pouvoirs seraient inopérants", ainsi traduit en italien «Ebbene sì, senza poteri l'amore non è niente / E senza amore il potere resterebbe inerte ». Nous avons essayé de préserver autant que possible les assonances de la langue de départ et la syntaxe de la langue d'arrivée. Dans ce cas particulier, l'italien offrait la possibilité d'insister non seulement sur les sifflantes, mais aussi sur les liquides provoquant un jeu continuel d'échos, d'amplifications et de renvois. Comme le souligne Meschonnic (Poétique 140), il est nécessaire de traduire ce que les mots font, l'agir même du langage, souvent si sournois que l'on ne s'en aperçoit guère. Ce n'est pas par hasard que le théâtre représente alors un domaine non seulement ancien mais aussi significatif, pour mettre en crise toute notion de sens et de rythme.

Le recours fréquent aux déictiques souligne l'importance des mouvements et des déplacements sur scène. Le français utilise également un grand nombre d'adjectifs qu'il fallait absolument respecter en traduisant. Ces adjectifs constituent en effet le meilleur moyen d'évaluation de l'«impasse » initiale et l'exaspération qui touche son comble dans la scène finale où «Elle se 
dévêt de sa tenue de prêtresse, de ses voiles de mariée et de son diadème / Elle les enduit de ses poisons les plus violents, les plus subtils et indécelables » qui donne en italien « Si toglie la tonaca da sacerdotessa, il velo da sposa e il diadema / Li cosparge dei veleni più potenti, perfidi e segreti ». L'accumulation souligne le pathos: Médée a lancé son anathème avec toute l'agressivité que les voix du mal l'ont poussée à manifester, et les sonorités qui l'accompagnent ne peuvent être alors que des dentales.

Pour ce qui est des pronoms personnels, l'on remarque leur utilisation dans les deux textes pour souligner la lutte titanesque de l'héroïne seule contre tous, bouc émissaire des innombrables manquements des autres, sorcière-étrangère abandonnée à son destin, mais qui malgré tout ne renonce jamais à son ego démesuré. Il prévaut aux côtés d'une troisième personne plurielle qui se manifeste aussi bien au féminin, les femmes du Chœur de Corinthe - qui en intervenant ne font qu'accroître la colère de Médée - qu'au masculin, les détenteurs du pouvoir illégitime. C'est ainsi qu'à la Scène 5, une Médée exaspérée finit par se dresser même contre Lyssa : «Assez Lyssa, tu sais bien que je n'ai tué personne / Et qu'elles ont tout inventé pour me marginaliser / Et mieux se complaire dans leurs compromis / Qui confortent des pouvoirs sans conventions / Et après, elles n'ont plus qu'à se taire et à subir / Quand leurs enfants le paient de leurs vies / Et de ça je devrais me sentir solidaire ? Jamais!», traduit en italien par: "Basta Lissa, sai bene che non bo ucciso nessuno / E che loro si sono inventate tutto per isolarmi / E abbandonarsi più facilmente ai loro compromessi / Che appoggiano poteri senza tradizioni / Dopo douranno soltanto subire e tacere / Quando i loro figli pagheranno con la vita / É forse questo che dovrei capire? Mai!». L'italien préserve donc l'utilisation des pronoms personnels et des adjectifs possessifs, afin de mettre en exergue une idée bien précise de possession et d'appartenance, dans le cadre d'une lutte sans merci.

Il a été également important de prendre en compte les impératifs, véhicules morphologiques de l'autorité de Médée, et ses phrases trop courtes ou incomplètes, qui se reflètent dans la succession rapide des réactions des antagonistes sur scène. L'enchevêtrement des renvois enrichit ces conversations savantes et l'on a volontairement choisi d'ennoblir le style de la langue d'arrivée, afin que le registre soit à la hauteur du mythe. D'un point de vue syntaxique, l'original présente plusieurs syntagmes nominaux et une utilisation assez limitée des subordonnées, cela afin de simplifier les phrases et de respecter la linéarité du vers. Etant donné la densité du texte, on a préféré ne pas ajouter de notes explicatives qui auraient interrompu le plaisir d'une lecture fluide.

Le traducteur se doit surtout de libérer les expressions apparemment floues de toute ambiguité, et d'éviter les pertes absolues ainsi que les transcriptions qui échapperaient à une compréhension. On ne trouvera qu'un seul cas de transcription dans le Chant de Lyssa (Sc.6) où Médée parle «argot» en créant une rime importante avec le nom du navire Argo. Toujours pour les mêmes raisons, l'on a eu recours à la modulation pour la version en langue d'arrivée dans le cas suivant, où l'on met l'accent plus sur le résultat que sur l'action : «Ah Médée que tout soit bien qu'avaistu à vouloir / Ne savais-tu pas que c'était se condamner à ne rien avoir? ?(Sc.4). En italien, la rime a été ainsi recréée: "Ah Medea come facevi a volere che tutto fosse perfetto / Non sapevi che ti condannavi così a un nulla di fatto? ». Dans certains cas, l'on a inévitablement essuyé des pertes, par exemple quand il s'agissait de rendre le vers : "C'était à elle la claire audience et la claire voyance » qui a été traduit par "Suoi erano il presentimento e la chiaroveggenza» (Sc.4). Ce faisant, la rime intérieure et la répétition de l'adjectif ont été perdues, ce qui a été cependant compensé par deux substantifs affichant des préfixes riches en connotations.

Comme la production collective se veut recherche, remise en cause permanente et méthode de travail qui privilégie le débat, la réflexion, la critique, il s'agit bien sûr d'une esthétique en devenir, toujours perfectible, mais qui nous témoigne que les dialogues entre les différentes disciplines artistiques et les nombreux créateurs peuvent s'épanouir. Comme le souligne Caya Makhélé (11), «Qu'il soit inspiré des contes ou issu d'un texte original dont le caractère contemporain n'est 
guère contestable, l'hétérogénéité est le ferment de ce théâtre ». Toute expérience artistique peut donc trouver dans ce type de théâtre un terrain fertile pour sa pleine réalisation vu la richesse ainsi que la multiplicité des formes et des langages adoptés.

Le but de l'écrivain semble être celui de secouer les consciences, pour conduire à une réflexion et à un dialogue constructifs. Il serait trop facile de fournir des solutions toutes faites de la scène. S'il existe des points de rencontre, parait suggérer l'auteur, au-delà des préjugés, des intégrismes idéologiques ou des schématismes disciplinaires, ceux-là ne deviendront effectifs qu'à travers un long et difficile parcours de connaissance, d'échange et de médiation entre des systèmes différents de valeurs, de formes et de langages. De là vient l'originalité de cet effort créatif qui a le regard tourné vers le passé, comme pour Eurydice, mais dans le but de préparer un futur meilleur à travers la réintégration des traditions ancestrales. L'artiste sait annuler les distinctions trop nettes, les règles trop rigides, en lançant ainsi un merveilleux défi à la pensée symbolique de l'homme contemporain.

Puisque ce théâtre embrasse tout entier le continent d'où il surgit, il arrive à occuper une position remarquable parmi les expressions de l'âme africaine et offre un brillant exemple de la dimension multiculturelle qui l'anime. Werewere-Liking arrive ainsi à créer une admirable aventure itinérante non seulement à travers l'amplification des espaces et des temps, mais aussi grâce à des musiques et à des couleurs envoûtantes. Bien que l'Afrique soit une terre démesurée, la compagnie Ki-Yi a pour but la recherche des constantes culturelles. Pour cela, nous assistons à la multiplication des techniques d'expression unies à un langage très singulier et à grand potentiel poétique. Le style de l'auteur révèle partout un rythme vital et en même temps aliénant.

Pour préparer notre traduction, nous avons dû étudier à fond toutes ces caractéristiques parce que les principes qui nous ont servi de guide ont été la fidélité au texte original avec la volonté d'en respecter et d'en reproduire au mieux la riche alchimie originale. Traduire signifie donc ici avoir une méthode rigoureuse, une organisation particulière et une certaine «spécialisation » sur le genre de texte à affronter. Considérer ce travail comme une tâche à remplir uniquement à l'écrit serait limitatif. Pour cela, il est fondamental que le traducteur lise et relise à haute voix son texte cible afin qu'il puisse un jour donner lui aussi sur scène le même enthousiasme que l'original. La traduction théâtrale est en effet un entrelacement de voix qui ne manquent pas de partir vers d'autres expérimentations et d'autres dialogues, afin de mettre en valeur le mouvement et de renouveler sans cesse les jeux de transparence et d'opacité. 


\section{Bibliographie}

Benelli, Graziano. "Riflessioni sulla traduzione verso l’italiano." Rivista internazionale di Tecnica della Traduzione 4 (1999) : 69-80.

Buffoni, Franco. "Leopardi in lingua inglese come paradigma della simbolicità del compito di un poeta traduttore." La tradurione poetica. Ed. AA.VV. Milano : Guerini e Associati, 1989. 91-112.

Eco, Umberto. Dire quasi la stessa cosa. Milano : Bompiani, 2003.

Froelich, Julienne. Animismes. Les religions païennes de l'Afrique de l'Ouest. Paris : Ed. de l'Orante, 1964.

Ladmiral, Jean-René. "Sourciers et ciblistes." Revue d’Esthétique 12 (1986) : 33-42.

Liking, Werewere. Marionnettes du Mali. Paris : NEA-Arhis, 1987.

---. Le Parler-Chanter, Parlare cantando. Torino : L'Harmattan Italia, 2003 (Introduction, Traduction et Note sur la traduction de Nataša Raschi).

---. Médée les risques d'une réputation / Medea i rischi d'una certa reputarione. Ed. Anna Paola Mossetto, Torino : Libreria Stampatori, 2006 (Traduction, Note sur la traduction et interview à l'auteur de Nataša Raschi).

Makhélé, Caya. "Les voix du théâtre contemporain en Afrique." Alternatives théâtrales 48 (juin 1995) : 5-15.

Meschonnic, Henri. Critique du rythme. Anthropologie historique du langage. Paris : Verdier, 1982.

---. Poétique du traduire. Lagrasse : Verdier, 1999.

Mossetto, Anna Paola. "La sfida africana di Medea." Magia, gelosia, vendetta. Il mito di Medea nelle lettere francesi. Ed. Liana Nissim et Alessandra Preda. Milano : Cisalpino, 2006. 379-391.

Mounin, Georges. Les problèmes théoriques de la traduction. Paris : Gallimard, 1976.

Pacéré, Titinga Frédéric. Le langage des tams-tams et des masques en Afrique. Paris : L'Harmattan, 1991.

Sidibé, Valy. "Théâtre populaire et langues nationales. Étude de cas en Côte d'Ivoire." Théâtres africains. Ed. AA.VV.. Paris : Silex, 1990. 97-103.

Tagliacozzo, Sara. "Postfazione. Questioni di reputazione. Il teatro antropologico di Werewere Liking". Médée les risques d'une réputation / Medea i rischi d'una certa reputazione. Ed. Werewere Liking. Torino : Libreria Stampatori, 2006. 130-135.

Vernant, Jean-Pierre, Vidal-Naquet, Pierre. Mythe et tragédie en Grèce ancienne. Paris : Maspéro, 1972. 